Journal of Molecular Structure, 162 (1987) 247-254

Elsevier Science Publishers B.V., Amsterdam - Printed in The Netherlands

\title{
THE MICROWAVE SPECTRUM AND STRUCTURE OF ALLYL ALCOHOL
}

\author{
HASSAN BADAWI, PRIMOŽ LORENČCAK, KURT W. HILLIG II, MISAKO IMACHI \\ and ROBERT L. KUCZKOWSKI \\ Department of Chemistry, The University of Michigan, Ann Arbor, MI 48109 (U.S.A.) \\ (Received 3 June 1987)
}

\begin{abstract}
The microwave spectrum of the normal species of the gauche, gauche isomer of allyl alcohol has been reassigned and rotational and centrifugal distortion constants have been determined. Rotational constants of nine deuterated species, three carbon-13 species and the oxygen-18 species have also been obtained. These data were used to estimate structural parameters by least-squares fitting of the effective moments of inertia. Good agreement with an earlier electron diffraction and ab initio study was obtained. Unassigned transitions from another isomer were observed, presumably the cis, gauche conformer.
\end{abstract}

\section{INTRODUCTION}

The conformation and structure of allyl alcohol has been the subject of considerable study using experimental and theoretical probes. This work was recently reviewed in a report deducing its molecular structure from electron diffraction, microwave and infrared data and geometry-related ab initio calculations [1]. In concurrence with prior experimental and theoretical work these investigators found evidence for two low energy conformers, namely the cis, gauche (or $\mathrm{sp},-\mathrm{sc}$ ) and the gauche, gauche (or -ac, sc) illustrated in Fig. 1. The ab initio (4-21G basis) calculations estimated the cis, gauche conformer to be lower by $0.54 \mathrm{kcal} \mathrm{mol}^{-1}$. The experimental analysis (primarily from ED data) estimated a conformer mixture of 57(6)\% cis, gauche and 43(6)\% gauche, gauche at roum temperature. Both ab initio and experimentally derived structural parameters were estimated for each conformer. The mixture of the two forms made the experimental analysis too complex to obtain independent structural parameters without some external constraints so that only 14 geometrical parameters were refined during the fitting process.

Until our study, only two papers on the MW spectrum of allyl alcohol have appeared. Murty and Curl assigned approximately 20 transitions $(J \leqslant 6)$ for the normal, ${ }^{18} \mathrm{O}$ and $\mathrm{OD}$ isotopic species of the gauche, gauche form [2]. Jaman and Ghosh have reported a centrifugal distortion analysis of 40 transitions for the normal species [3]. 

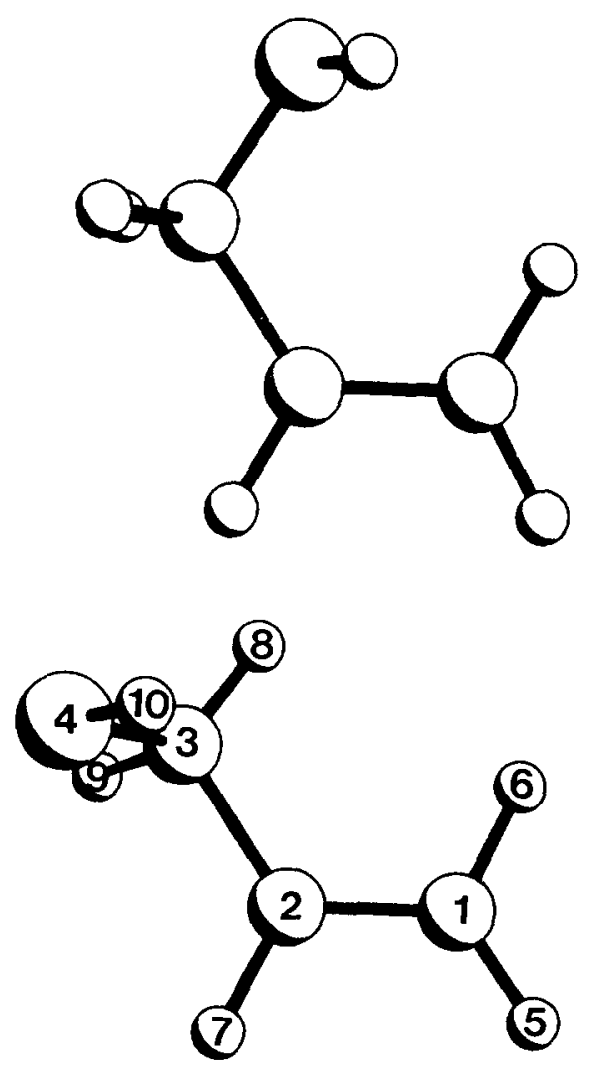

Fig. 1. Low energy conformers of allyl alcohol [1]. Top, cis, gauche (sp, -sc); bottom, gauche, gauche (-ac, sc).

During catalytic studies of the conversion of allyl alcohol to acrolein $[4,5]$, we prepared several deuterated samples of allyl alcohol and assigned their $R$ branch, a dipole spectra but did not publish the spectroscopic details. This start prompted us to assign the additional isotopic species necessary to determine structural parameters from spectroscopic constants alone, an undertaking which commenced before the recent diffraction report. This paper reports these results for the gauche, gauche conformer. It also became clear that a number of the high $J$ transitions for this conformer in the earlier study [3] were misassigned so that a complete study of available high $J$ lines in the region $26.5-40.0 \mathrm{GHz}$ was undertaken for the normal species. This also seemed valuable in order to ascertain which transitions, if any, may arise from another isomer. This will be discussed subsequently.

\section{EXPERIMENTAL}

The spectra were primarily recorded with a Hewlett-Packard 8460 A spectrometer in the region of $26.5-40.0 \mathrm{GHz}$. Frequency measurements were 
made by locating line centers on chart recorder tracings and from computer controlled runs with a CRT display. The latter mode of operation allowed signal averaging techniques to be used. Transitions were also measured between 18.0 and $26.5 \mathrm{GHz}$ but this region was not systematically explored for all the isotopes. The $a$ dipole $J=0 \rightarrow 1$ and $1 \rightarrow 2$ transitions for the normal, the three ${ }^{13} \mathrm{C}$ isotopic species (natural abundance, 1.1\%) and several deuterium enriched species $\left(D_{8}, D_{9}\right.$ and $\left.D_{8,9}\right)$ were measured in the region of 8-18 GHz using a recently constructed Fourier transform spectrometer employing a Fabry-Perot cavity $[6,7]$. A supersonic molecular beam prepared from the saturated vapor pressure of allyl alcohol at room temperature in about 1 atm of argon was pulsed into the vacuum chamber resulting in very low rotational temperatures. The ${ }^{13} \mathrm{C}$ transitions could be observed after approximately 500-1000 microwave pulses although frequency measurements were made after considerably longer signal averaging ( $\sim 20000$ pulses). Stark effects, intensities, the frequency fits and reasonable consistency among the well determined centrifugal distortion constants were the criteria used to assign transitions. RF-MW double resonance techniques were used to locate and confirm the nearly degenerate $K_{\mathrm{p}}=2, J=3 \rightarrow 4$ transitions for all the isotopic species as well as some higher $J$ transitions for the normal, $3{ }^{13} \mathrm{C}$ and $9-d$ species. Our measurements for the normal, ${ }^{18} \mathrm{O}$ and OD species usually agreed to within $\pm 1 \mathrm{MHz}$ with the initial study [1]. The assignment of the transitions in the later study [2] with $J \geqslant 9$ is incompatible with our new assignments which show an order of magnitude better agreement between $\nu_{\text {obs }}-v_{\text {calc }}$.

The synthesis of the $6-d$ species in $70 \%$ enrichment from propargyl alcohol, $\mathrm{LiAlH}_{4}$ and $\mathrm{D}_{2} \mathrm{O}$ is described in our earlier study [5]. A similar technique using $\mathrm{LiAlD}_{4}$ and $\mathrm{D}_{2} \mathrm{O}$ [8] was employed to obtain a mixture of the $6,7-d_{2}(68 \%)$ and $7-d(30 \%)$ species. The reaction of propargyl alcohol and $\mathrm{D}_{2}$ catalyzed by $\mathrm{Pd} / \mathrm{BaSO}_{4}$ led to $5-d(21 \%), 7-d(23 \%), 5,7-d(37 \%)$ and the normal species [9]. While the $7-d$ and $5,7-d_{2}$ species were extensively assigned, only the $\mu_{\mathrm{a}} R$ branch transitions were assigned for the $5-d$ species before the sample was depleted. The $8,9-d_{2}$ species was prepared in high enrichment from acryloyl chloride and $\mathrm{LiAlD}_{4}[10]$. When a mixture of $\mathrm{LiAlH}_{4}$ and $\mathrm{LiAlD}_{4}$ was employed the 8-d and 9-d species along with the $8,9-d_{2}$ and the normal species were obtained in approximately equal abundance. The OD sample was obtained by exchange with $\mathrm{D}_{2} \mathrm{O}$. The preparation of the ${ }^{18} \mathrm{O}$ species ( $\sim 20 \%$ enriched) is described in the earlier study [5]. The $1-{ }^{13} \mathrm{C}$ species was prepared by reacting vinylmagnesium bromide with $99 \%{ }^{13} \mathrm{CH}_{2} \mathrm{O}$ (MSD isotopes) [11]. The $3{ }^{13} \mathrm{C}$, and $2-{ }^{13} \mathrm{C}$ species were detected in natural abundance but only the $K=2, J=3 \rightarrow 4$ and $0 \rightarrow 1$ and $1 \rightarrow 2$ transitions could be unambiguously assigned by using the RF-MW or FT-MW techniques discussed above. 


\section{STRUCTURE DETERMINATIONS}

The rotational and centrifugal distortion constants for the 11 species listed in Table 1 were determined with the program ZFAP6 written by Dr. V. Typke of the University of Ulm. Representation $I^{\mathrm{r}}$ and the Typke/van Eijck centrifugal distortion parameters were chosen $[12,13]$. The rotational constants for the species in Table 2 were determined with the five 4th order constants fixed at the values for the normal species. The transition frequencies are given in supplementary tables 1S-13S deposited with the British Library Lending Division as Supplementary Publication No 26343 (16 pages).

Allyl alcohol has 24 unique structural parameters. Although there are 39 well determined rotational constants, it is not possible to determine Kraitchman single substitution coordinates [14] for every atom due to the unavailability of reliable $A$ rotational constants for 3 species. The atomic coordinates were instead determined by least-squares fitting of the 39 experimental moments of inertia with equal weights. The program STRFIT7 was used which is a revised version of the structure fitting program discussed by Schwendeman [14]. The coordinates obtained are listed in Table 3. The RMS deviation of the fit was 0.0038 a.m.u. $\AA^{2}$. The derived structural parameters are given in the second column of Table 4 . The coordinates from this fit agreed within $0.005 \AA$ with those calculated from Kraitchman's equations except for $a$ of $\mathrm{C}_{3}$, and $a$ and $c$ of $\mathrm{H}_{8}$ which disagreed by -0.016 , -0.016 and $0.008 \AA$ respectively.

Inspection of these structural parameters indicates that parameters associated with $\mathrm{H}_{8}$ might be considered to deviate a little beyond the typical values. Also, the torsional angles involving the $\mathrm{CH}_{2}=\mathrm{CH}-\mathrm{C}$ moiety deviate a few degrees from planarity. In order to examine how the least squares fitting adjusts other parameters if $\mathrm{C}_{3}-\mathrm{H}_{8}$ is constrained to $1.095 \AA$ and the vinyl group to planarity, a second structure is provided in Table 4 . The RMS deviation of this fit was 0.0086 a.m.u. $\AA^{2}$. The differences between these two structures can be taken as an indication of the uncertainty in the $r_{0}$ structure (no assump.; see footnote to Table 4) arising in part from the many small coordinates. Nevertheless, the comparison in Table 4 between these essentially $r_{0}$ parameters and the $r_{\mathrm{g}}$ values determined either by electron diffraction or by ab initio techniques is generally good except for some of the angles associated with the $\mathrm{CH}_{2} \mathrm{OH}$ moiety where differences of $2-3^{\circ}$ occur. The $\mathrm{C}_{2}-\mathrm{C}_{3}-\mathrm{O}_{4}-\mathrm{H}_{10}$ torsional angle also is 4 to $6^{\circ}$ smaller. This is probably as good an agreement as can be obtained without making vibrational corrections to convert the spectroscopic constants from effective values $\left(A_{0}\right.$, etc.) to average values $\left(A_{z}\right.$, etc. $)$ with a similar reworking of the diffraction data. This was not undertaken since the spectroscopic corrections are difficult to estimate due to a lack of information on the effects of isotopic substitution on the average structure [15]; also the new insights to be gained from such an effort seemed marginal at this time. Of course the 


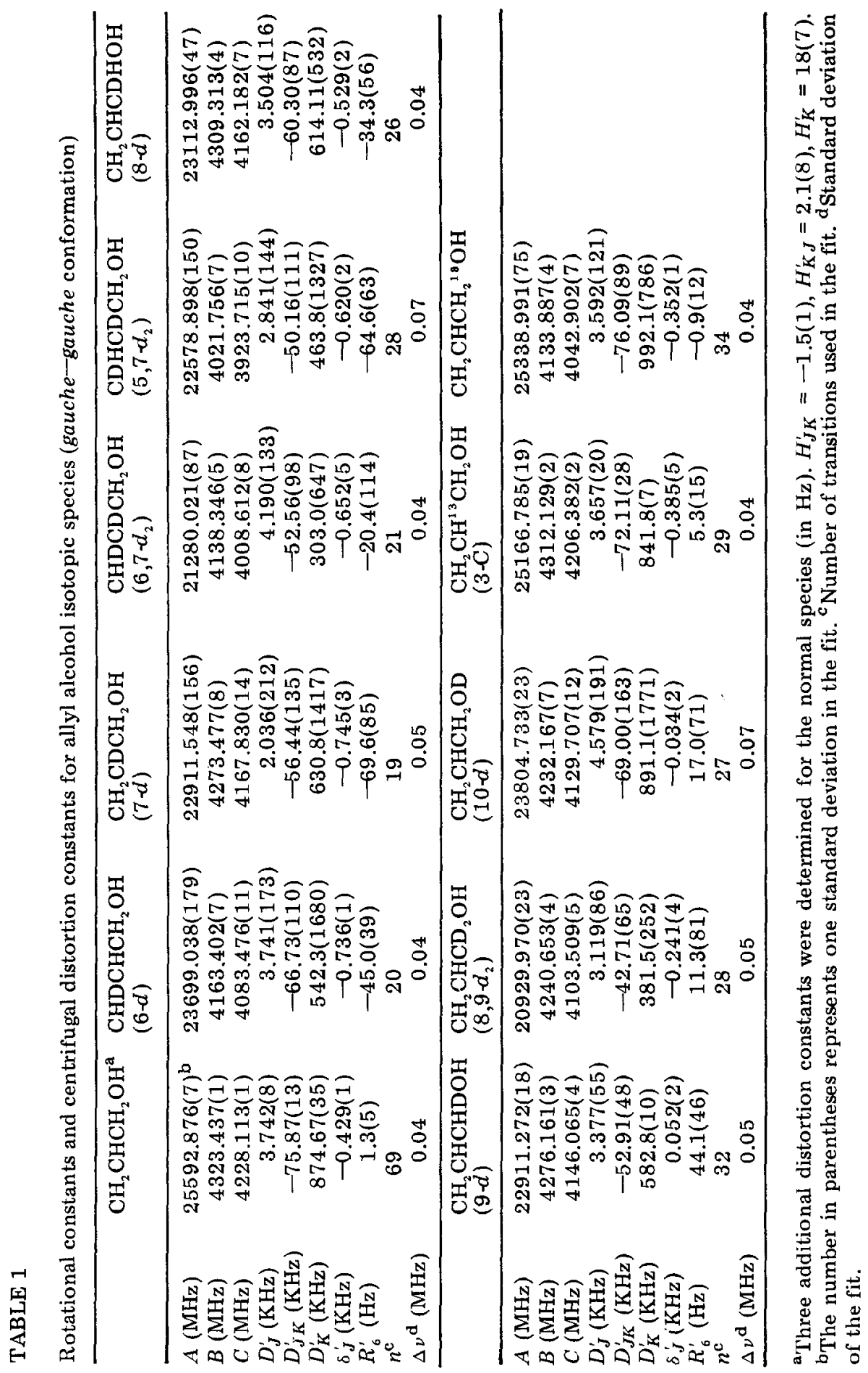


TABLE 2

Rotational constants for allyl alcohol isotope species (gauche-gauche conformation); centrifugal distortion constants fixed at values for normal species

\begin{tabular}{llll}
\hline & $\begin{array}{l}\mathrm{CDHCHCH}_{2} \mathrm{OH} \\
(5-d)\end{array}$ & $\begin{array}{l}{ }^{13} \mathrm{CH}_{2} \mathrm{CHCH}_{2} \mathrm{OH} \\
(1-\mathrm{C})\end{array}$ & $\begin{array}{l}\mathrm{CH}_{2}{ }^{13} \mathrm{CHCH}_{2} \mathrm{OH} \\
(2-\mathrm{C})\end{array}$ \\
\hline$B(\mathrm{MHz})$ & $4067.453(55)$ & $4201.267(15)$ & $4299.791(36)$ \\
$C(\mathrm{MHz})$ & $3971.934(55)$ & $4113.608(20)$ & $4209.864(36)$ \\
$n^{\mathrm{a}}$ & 8 & 7 & 6 \\
$\Delta \nu^{\mathrm{a}}(\mathrm{MHz})$ & 0.04 & 0.07 & 0.10 \\
\hline
\end{tabular}

${ }^{a}$ Number of transitions and standard deviations of the fit.

\section{TABLE 3}

Principal axes coordinates $(\AA)$ for gauche-gauche allyl alcohol from least-squares fitting of all moments of inertia ${ }^{a}$, center of mass and principal axis conditions

\begin{tabular}{lrrr}
\hline & \multicolumn{1}{c}{$a$} & \multicolumn{1}{c}{$b$} \\
\hline $\mathrm{C}_{2}$ & 1.8384 & 0.0528 & -0.2727 \\
$\mathrm{C}_{2}$ & 0.7021 & 0.1891 & 0.4094 \\
$\mathrm{C}_{3}$ & -0.5612 & -0.5724 & 0.1206 \\
$\mathrm{O}_{4}$ & -1.6567 & 0.2923 & -0.1596 \\
$\mathrm{H}_{5}$ & 1.8874 & -0.6738 & -1.0965 \\
$\mathrm{H}_{6}$ & 2.7226 & 0.6206 & -0.0601 \\
$\mathrm{H}_{7}$ & 0.6253 & 0.9264 & 1.2199 \\
$\mathrm{H}_{8}$ & -0.2958 & -1.2779 & -0.7135 \\
$\mathrm{H}_{6}$ & -0.8201 & -1.1509 & 1.0140 \\
$\mathrm{H}_{10}$ & -1.3939 & 0.8550 & -0.8945 \\
\hline
\end{tabular}

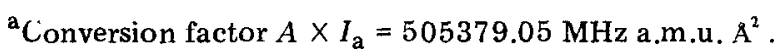

physical meaning of the $r_{0}$ structure and error estimates are ambiguous. We believe that if the differences between the two spectroscopic structures in Table 4 are doubled, then the values in column 2 with these attached uncertainties should encompass the well defined average structure $\left(r_{\mathrm{z}}\right)$.

\section{DISCUSSION}

This study of the isotopic species of allyl alcohol is in essential agreement with the structural analysis by electron diffraction and ab initio techniques. The ED analysis was a complex deconvolution performed with scattering data from a mixture of two conformers, spectroscopic constants from one species and constraints partly obtained from ab initio calculations. Only 14 geometrical parameters for the two conformers were fitted. The basic consistency between the structural details from thal study and the spectroscopic results is satisfying. This agreement also lends confidence in the 
TABLE 4

Comparison of structural parameters for gauche-gauche allyl alcohol ${ }^{\mathrm{a}}$

\begin{tabular}{|c|c|c|c|c|}
\hline & \multicolumn{2}{|c|}{ Spectroscopic ${ }^{\mathrm{b}}$} & \multirow[t]{2}{*}{$\mathrm{ED}^{\mathrm{c}}$} & \multirow[t]{2}{*}{ Theord } \\
\hline & No Assump. & Planar Assump. & & \\
\hline$C_{1}=C_{2}$ & 1.332 & 1.337 & 1.336 & 1.340 \\
\hline $\mathrm{C}_{2}-\mathrm{C}_{3}$ & 1.503 & 1.502 & 1.495 & 1.500 \\
\hline $\mathrm{C}_{3}-\mathrm{O}_{4}$ & 1.423 & 1.428 & 1.432 & 1.430 \\
\hline $\mathrm{C}_{1}-\mathrm{H}_{5}$ & 1.072 & 1.078 & 1.101 & 1.107 \\
\hline $\mathrm{C}_{1}-\mathrm{H}_{6}$ & 1.099 & 1.091 & 1.103 & 1.108 \\
\hline $\mathrm{C}_{2}-\mathrm{H}_{7}^{\circ}$ & 1.098 & 1.092 & 1.101 & 1.108 \\
\hline $\mathrm{C}_{3}^{2}-\mathrm{H}_{8}$ & $1 . \mathrm{i} 24$ & 1.096 & 1.110 & 1.118 \\
\hline $\mathrm{C}_{3}-\mathrm{H}_{9}$ & 1.095 & 1.102 & 1.106 & 1.114 \\
\hline $\mathrm{O}_{4}-\mathrm{H}_{10}$ & 0.962 & 0.960 & 1.029 & 0.967 \\
\hline $\mathrm{O}_{3}-\mathrm{C}_{2}-\mathrm{C}_{1}$ & 124.5 & 123.9 & 125.3 & 124.2 \\
\hline $\mathrm{O}_{4}-\mathrm{C}_{3}-\mathrm{C}_{2}$ & 112.1 & 111.8 & 112.2 & 111.4 \\
\hline $\mathrm{H}_{5}-\mathrm{C}_{1}-\mathrm{C}_{2}$ & 123.2 & 122.0 & 119.9 & 122.0 \\
\hline $\mathrm{H}_{6}-\mathrm{C}_{1}-\mathrm{C}_{2}$ & 119.3 & 119.8 & 119.9 & 122.0 \\
\hline $\mathrm{H}_{7}-\mathrm{C}_{2}-\mathrm{C}_{1}$ & 120.4 & 121.0 & 118.9 & 121.0 \\
\hline $\mathrm{H}_{3}-\mathrm{C}_{3}-\mathrm{C}_{2}$ & 105.2 & 107.7 & 111.9 & 110.6 \\
\hline $\mathrm{H}_{3}-\mathrm{C}_{3}-\mathrm{O}_{4}$ & 114.6 & 113.3 & 112.3 & 111.0 \\
\hline $\mathrm{H}_{9}-\mathrm{C}_{3}-\mathrm{C}_{2}$ & 108.0 & 108.0 & 112.1 & 110.8 \\
\hline $\mathrm{H}_{9}-\mathrm{C}_{3}-\mathrm{O}_{4}$ & 107.4 & 107.3 & 105.8 & 104.5 \\
\hline $\mathrm{H}_{10}-\mathrm{O}_{4}-\mathrm{C}_{3}$ & 107.2 & 107.3 & 102.8 & 109.7 \\
\hline $\mathrm{C}_{1} \mathrm{C}_{2} \mathrm{C}_{3} \mathrm{O}_{4}$ & -122.0 & -122.9 & -122.0 & -128.0 \\
\hline $\mathrm{C}_{2} \mathrm{C}_{3} \mathrm{O}_{4} \mathrm{H}_{10} \mathrm{e}$ & 56.3 & 55.9 & 62.0 & 60.0 \\
\hline
\end{tabular}

"Parameters in $A$ and degrees. "bo Assumption" is a fit to inertial equations only; "Planar Assump." is a fit to inertial equations (weight $=1$ ), $d\left(\mathrm{C}_{3}-\mathrm{H}_{8}\right)=1.095$ (weight = 10) and angle assumptions (weight $=20$ ) for the $\mathrm{CH}_{2}=\mathrm{CHC}$ atoms which constrain this moiety to planarity. ${ }^{c}$ Electron diffraction determination, $r_{\mathrm{g}}$ parameters, ref. $1 .{ }^{\mathrm{d}} \mathrm{Ab}$ initio values, $r_{\mathrm{g}}$ (4-21G basis and appropriate corrections), ref. 1 . ${ }^{\mathrm{e}}$ Other torsional angles which show the deviation of the $\mathrm{CH}_{2}=\mathrm{CHC}$ moiety from planarity: $\mathrm{H}_{3}-\mathrm{C}_{1}-\mathrm{C}_{2}-\mathrm{H}_{6}=$ 180.0, $\mathrm{C}_{3}-\mathrm{C}_{2}-\mathrm{C}_{1}-\mathrm{H}_{5}=178.8, \mathrm{H}_{5}-\mathrm{C}_{1}-\mathrm{C}_{2}-\mathrm{H}_{7}=1.5$. The convention for torsional angles is taken from ref. 16 .

parameters estimated for the cis-gauche isomer ( $\mathrm{sp}, \mathrm{-sc})$ in ref. 1.

In the analysis of the spectrum of the normal species, virtually every strong transition was assigned for the gauche, gauche isomer between 26.5 and $40.0 \mathrm{GHz}$. It was apparent that approximately 100 additional strong lines remain unassigned which arise from another isomer. Temperature dependence studies were inconclusive in determining whether the unassigned conformer was lower in energy. The ED and ab initio results indicate that the cis, gauche conformer is slightly lower in energy and the reported structural parameters should assist the MW analysis; however, efforts at assigning the transitions have been unsuccessful. Both the Stark modulated spectrum between 26.5 and $40.0 \mathrm{GHz}$ and the FTMW spectrum between 8 and $12 \mathrm{GHz}$ have been examined. The latter spectral searches employed a pulsed nozzle 
and have also led to several transitions presumably arising from the cis, gauche form. We suspect that the transitions from the cis, gauche form may be complicated by $\mathrm{OH}$ tunneling which has hindered their assignment. Work is continuing in order to assign them.

\section{ACKNOWLEDGMENT}

The authors are grateful to the donors of the Petroleum Research Fund, administered by the American Chemical Society for the support of this work in the form of a fellowship to M.I. The work has also been supported by grants CHE-8303615 and 8603834 from the National Science Foundation, together with grants 8305806 and 8614340 which funded the construction of the FTMW spectrometer. We have appreciated the interest in this work by Professor Lothar Schäfer which has stimulated its progress. We gratefully acknowledge the help of Dr. Marabeth LaBarge in confirming some assignments with the FTMW spectrometer.

\section{REFERENCES}

1 F. Vanhouteghem, W. Pyckhout, C. Van Alsenoy, L. Van Den Enden and H. J. Giese, J. Mol. Struct., 140 (1986) 33.

2 A. N. Murty and R. F. Curl, Jr., J. Chem. Phys., 46 (1967) 4176.

3 A. I. Jaman and D. K. Ghosh, Pramana, 17 (1981) 245.

4 M. Imachi, N. W. Cant and R. L. Kuczkowski, J. Catal., 75 (1986) 404.

5 H. S. Choi, J. T. Lin and R. L. Kuczkowski, J. Catal., 99 (1986) 72.

6 T. J. Balle and W. H. Flygare, Rev. Sci. Instrum., 52 (1981) 33.

7 K. W. Hillig, II, J. Matos, A. Scioly and R. L. Kuczkowski, Chem. Phys. Lett., 133 (1987) 359.

8 E. B. Bates, E. R. H. Jones and M. C. Whiting, J. Chem. Soc., (1954) 1854.

9 A. I. Vogel, A Textbook of Practical Organic Chemistry, 3rd edn., Wiley, New York, 1956, p. 951.

10 R. D. Schnetz and F. W. Millard, J. Org. Chem., 24 (1959) 297.

$11 \mathrm{~K}$. Nützel, Methoden Zur Herstellung und Umwandlung Magnesiumorganischer Verbindungen, in E. Müller, O. Bayer, H. Meervein and K. Ziegler (Eds.), Methoden der Organischen Chemie, Vol. XIII/2a, Georg Thieme Verlag, Stuttgart, 1973, p. 289.

12 V. Typke, J. Mol. Spectrosc., 53 (1976) 170.

13 B. P. van Eijck, J, Mol. Spectrosc., 63 (1974) 246.

14 R. H. Schwendeman, in D. R. Lide and M. A. Paul (Eds.), Critical Evaluation of Chemical and Structural Information, National Academy of Sciences, Washington DC, 1974 , pp. $74-115$.

15 K. Kuchitsu, T. Fukuyama and Y. Morino, J. Mol. Struct., 4 (1969) 41.

16 E. B. Wilson, Jr., J. C. Decius and P. C. Cross, Molecular Vibrations, McGraw-Hill, New York, 1955. 